\title{
Philosophiques
}

\section{L’axiologie d'Ingarden}

\section{Victor Kocay}

Volume 22, numéro 1, printemps 1995

URI : https://id.erudit.org/iderudit/027307ar

DOI : https://doi.org/10.7202/027307ar

Aller au sommaire du numéro

Éditeur(s)

Société de philosophie du Québec

ISSN

0316-2923 (imprimé)

1492-1391 (numérique)

Découvrir la revue

Citer cet article

Kocay, V. (1995). L’axiologie d'Ingarden. Philosophiques, 22(1), 35-52.

https://doi.org/10.7202/027307ar

\section{Résumé de l'article}

Cet article met en lumière la formation des valeurs esthétiques entendues au sens de la philosophie de Roman Ingarden. L'auteur présente et commente trois notions essentielles de cette pensée : il s'agit de la concrétisation, de la valeur esthétique et du noyau de l'objet esthétique. Bien que la critique s'intéresse depuis longtemps à l'axiologie ingardénienne, la perspective de l'auteur, mettant en valeur la notion de noyau esthétique, indique clairement que la pensée d'Ingarden chevauche deux approches différentes en ce qui concerne la formation des valeurs. Lorsqu'il s'agit de la création de nouvelles valeurs, par exemple, la soi-disant objectivité de la philosophie d'Ingarden semble masquer le côté subjectif de leur formation.
Ce document est protégé par la loi sur le droit d'auteur. L'utilisation des services d'Érudit (y compris la reproduction) est assujettie à sa politique d'utilisation que vous pouvez consulter en ligne.

https://apropos.erudit.org/fr/usagers/politique-dutilisation/ 


\title{
L'AXIOLOGIE D'NGGRDEN
}

\author{
Victor Kocay
}

RÉSUME : Cet article met en lumière la formation des valeurs esthétiques entendues au sens de la philosophie de Roman Ingarden. L'auteur présente et commente trois notions essentielles de cette pensée : il s'agit de la concrétisation, de la valeur esthétique et du noyau de l'objet esthétique. Bien que la critique s'intéresse depuis longtemps à l'axiologie ingardénienne, la perspective de l'auteur. mettant en valeur la notion de noyau esthétique, indique clairement que la pensée d'Ingarden chevauche deux approches différentes en ce qui concerne la formation des valeurs. Lorsquil s'agit de la création de nouvelles valeurs, par exemple, la soi-disant objectivite de la philosophie d'Ingarden semble masquer le côté subjectif de leur formation.

\begin{abstract}
The objective of this article is first to present the major notions of Ingarden's philosophy with respect to axiology: concretization, aesthetic value and the core of the aesthetic object, and second, to show that Ingarden's so-called objective aesthetics covers up or masks the more subjective elements of value theory. Critics have long been interested in Ingarden's aesthetics, but the author's perspective, insisting on the importance of the core of the aesthetic objet, clearly reveals that Ingarden's thought actually involves two different approaches in aesthetic value theory, an empirical approach, and a more subjective, relative one which seems to remain hidden.
\end{abstract}

Dans cet article nous présentons la conception ingardénienne des valeurs esthétiques, et nous montrons que cette conception peut induire en erreur en ce qui concerne l'ontologie de ces valeurs. Nous proposons, mais sans pouvoir l'expliciter entièrement, une formulation autre à la fois plus juste et plus claire de la problématique de ces valeurs. La poussée ontologique de sa pensée aurait mené Ingarden à délimiter et à démarquer de façon objective - ćest-à-dire comme s'il s'agissait de plusieurs objets différents que le philosophe se donne pour but de distinguer les uns par rapport aux autres - ce qui ne constitue finalement que la perspective du philosophe, une perspective qui en esthétique est subjective et changeante. Il est donc possible de schématiser la pensée 
d'Ingarden afin de faire ressortir les distinctions par exemple entre les qualités et les valeurs : la valeur étant pour ainsi dire greffée sur les qualités de l'œuvre bien que le passage de la qualité à la valeur reste entouré de mystère dans cette philosophie. Dans le contexte d'un acte de lecture, c'est-à-dire l'expérience d'un lecteur qui lit une œuvre particulière, cette conception des valeurs esthétiques semble plutôt factice. A notre sens Ingarden comprend la réalisation des valeurs esthétiques de façon trop linéaire de sorte qu'il sous-estime le rôle du consommateur des objets d'art dans la constitution de ces valeurs.

Afin de faire valoir notre lecture d'Ingarden nous présentons et commentons ici les trois notions essentielles à l'analyse du texte littėraire dans sa philosophie, celle de concrétisation, celle de valeur esthétique, et celle du noyau de l'objet esthétique. Or, parler de l'objectivité des valeurs esthétiques exige une définition préalable de l'objectif. Nous nous servons de celle donnée par E. Fink dans son livre, De la phénoménologie, selon laquelle «objectif » veut dire « valide [...] pour tout le monde $»^{I}$. Cette définition nous paraît d'autant plus valable qu'Ingarden et Fink appartiennent tous deux, malgré leurs différences, à une même tradition philosophique fla phénoménologiel, et surtout que la pensée d'Ingarden porte sur l'ontologie, Cest-à-dire sur l'étude de l'être en tant qu'être. Un des objectifs principaux d'Ingarden est de « rẻsoudre » le conflit autour de l'ídéalisme et du réalisme. Il propose une notion d'objet d'une certaine envergure, étendue jusqu'a l'inclusion de l'objet de pensée. Se donner au préalable une définition empirique de l'objectif en sorte que la notion d'objet soit limitée à l'existence et au monde physique, c'est en un sens renier en un seul trait toute la philosophie d'Ingarden. La définition de Fink correspond bien aux notions d'Ingarden parce qu'elle est générale et concise, et qu'elle représente une ouverture nècessaire à la comprèhension de l'ontologie ingardénienne des valeurs.

\section{La concrétisation}

Notre point de départ est la notion ingardénienne de concrétisation ${ }^{2}$. Le terme de concrétisation désigne la constitution du référent de l'œuvre

I. E. Fink, De la phénoménologie, traduit par Didier Franck, Paris, Eđitions de Minuit, 1974, p. $29-30$.

2. La notion de concrétisation est métaphorique. Elle a pour contenu la façon dont le lecteur comprend le texte, c'est-à-dire qu'elle signifie le rapport entre le signe et son référent. Pourtant, le signifié désigné par le signifiant ne se «concrétise » jamais : il reste toujours sur le plan conceptuel et ne se revêt de traits « concrets » et « réels » que dans la conscience du lecteur. Il est vai que le lecteur « remplit » pour ainsi dire les lieux d'indétermination du texte, mais même cette façon de comprendre la notion de concrétisation est métaphorique. Le signifié ainsi « rempli » est tout aussi conceptuel que le signifié non concrétisé. 
littéraire dans la conscience du lecteur. En lisant un texte littéraire je me représente à moi-même - souvent de façon vague, soit - les personnages de l'œuve et leurs activités; je peux me représenter un paysage aussi bien que la situation dans laquelle un personnage se trouve. Je pourrais, par exemple, décrire le paysage dont parle un texte et juger de sa beauté, en disant à un ami que le paysage décrit dans cette œuvre est très joli, mais sans avoir jamais vu le paysage reel correspondant.

La concrétisation ne se limite pourtant pas à la représentation chez le lecteur du référent d'une œuvre; elle implique la participation active du lecteur au texte lu, le lecteur s'évertuant même à s'imaginer le référent des passages les plus obscurs.

Simplement dit, en lisant le lecteur remplit les lieux d'indétermination du texte. Si un roman cornmence par la phrase, «Pierre s'est levé à cinq heures », je sais que Pierre est un personnage, sans doute un être humain, un homme en particulier, et j'estime, sans avis contraire, que Pierre a un certain nombre d'annees, une volonté particulière, des émotions, des désirs, tout comme des êtres humains que je connais personnellement et véritablement. Si la deuxième phrase m'indique que Pierre a peigné ses cheveux noirs en se levant, je peux ajouter à ma concrétisation du personnage la couleur des cheveux et le fait que Pierre a des cheveux, mais n'ayant aucun renseignement ni sur la coupe ni sur le port de ses cheveux (des lieux d'indètermination), je remplis ces traits vides, même si ce n'est que de façon intuitive, selon mes propres goûts, peut-être selon mes souvenirs d'un ancien ami qui s'appelait lui aussi Pierre, ou peut-être selon mes connaissances de la mode en coiffure masculine de l'époque où a lieu l'histoire.

Le texte littéraire présente, d'ailleurs, la nècessitè des lieux indéterminés, et le lecteur les remplit selon ses capacités ou au moins jusqu'à ce qu'il comprenne le texte qu'il est en train de lire, et parfois jusqu'à proposer une lecture originale de l'œuvre. Toujours est-il que l'emprise de la réalité sur notre pensée est si grande qu'on veut faire même des objets fictionnels des êtres qui obéissent à notre sentiment du vrai et du réel. Dans la philosophie esthéticoontologique d'Ingarden cette activité de la part du lecteur qui remplit un texte, qui en constitue le référent, est désignée par le terme de concrétisation.

De la notion de concrétisation découle deux autres notions qui nous intéressent, la première va de soi, la deuxième est plus importante. (I) Il faut reconnaitre que l'objectivité absolue est un mythe en ce qui concerne la concrétisation d'un texte : on ne pourrait jamais arriver à formuler une interprétation qui serait la seule possible ou la seule qui soit scientifique. Puisque le lecteur s'implique dans sa lecture, il s'implique aussi dans l'interprétation qu'il donne de sa lecture, et chaque lecteur va comprendre le texte à sa façon, selon sa propre concrétisation. (2) En revanche, la notion de concretrisation 
exige une distinction d'ordre conceptuel entre l'œuvre en tant que telle et l'œuvre en tant que lue, ou bien, selon la terminologie d'Ingarden, entre l'objet artistique (l'œuvre) et l'objet esthétique (la concrétisation de l'œuvre). Deux personnes qui lisent un même roman en font chacune une concrétisation différente.

Or, une fois que l'on accepte la notion de concrétisation comme valide (ce qui nous semble préférable à la démarche contraire), il faut reconnaître que l'œuvre elle-même en tant qu' objet littéraire ne correspond jamais aux diverses concrétisations que différents lecteurs pourraient en faire, d'où la notion d'objet esthétique. L'expression « objet esthtétique » désigne chez Ingarden la concrétisation d'une ouvre littéraire chez un lecteur particulier. Les termes d'objet d'art et d'objet esthétique ne sont donc pas synonymes. L'objet d'art correspond a l'objet physique du monde tandis que l'objet esthétique est le résultat d'un effort intellectuel et d'une appréciation des qualités de l'objet artistique, objet qui en quelque sorte reste neutre dans la philosophie d'Ingarden.

Une question se pose pourtant : l'objet artistique et l'objet esthétique étant distincts l'un de l'autre, en quoi exactement diffèrent-ils? Chez Ingarden la réponse à cette question prend une formulation ontologique. L'objet artistique littéraire est inscrit dans les phrases imprimées d'un live, tandis que l'objet esthétique se formule dans la conscience du lecteur. Cette différence d'ordre ontologique constitue à notre sens la raison même pour laquelle certains chercheurs rejettent la notion d'objet esthétique d'après Ingarden, disant que cette notion fait de la lecture d'une œuvre littêraire un événement subjectif. D’après ceux qui s'opposent à cette notion, telle que nous la présentons, toute vérité « objective » serait impossible une fois que l'on accepte pour vraie l'existence de l'objet esthétique. Comment pourrait-on fonder l'objectif dans le cas d'un objet de pensée inaccessible à la pensée des autres, et difficile à saisir pour le sujet lui-même?

D'après Ingarden, cependant, l'objet esthétique n'existe pas en tant que tel; il n'est que reconnu existentiellement. Or, nous avouons ne pas bien saisir la différence entre « exister » et « être reconnu existentiellement ». Cette distinction semble provenir du souci toujours présent dans l'œuvre d'Ingarden d'éviter ce qu'il croyait être l'idéalisme de Husserl. Ainsi Ingarden ne voulait pas (pour ne pas, d'après nous, se laisser entraîner par la pensée idéaliste) affirmer l'existence de l'objet esthétique, prêférant en parler dans des termes ambigus. Nous préférons dire, par contre, que l'objet esthétique en fait existe. Evidemment il n'existe pas de la même manière que l'objet artistique, tout comme une pensée existe mais d'une façon autre qu'un rocher ou un arbre, d'une façon autre qu'un triangle équilatėral dans les théorèmes du géomètre. Des lectures diverses et parfois inconciliables d'une même œuvre semblent 
justifier la distinction de l'objet artistique de l'objet esthètique, ce qui semble également indiquer et que l'objet esthétique existe, mais toujours dans une concrétisation, et que chaque concrétisation est unique ${ }^{3}$.

Or, dire que l'objet esthétique existe, c'est se proposer un nouvel objet de recherche, constitué lors de la lecture de l'œuvre littéraire au fur et à mesure que le lecteur se représente le référent de l'œuvre en remplissant comme bon lui semble les lieux d'indétermination. Certaines qualités de l'œuvre attirent l'attention du lecteur de sorte que certaines valeurs esthétiques sont réalisées. Par exemple, la description heureuse d'un paysage constitue une valeur esthétique, le lecteur se plaisant à lire et à relire cette description, que ce soit à cause des mots utilisés, à cause de leur enchaînement, ou à cause de leurs propriétés évocatrices et ainsi de suite. De même, la représentation du comportement d'un certain personnage pourrait nous attirer pour des raisons linguistiques ou stylistiques, ou bien pour des raisons psychologiques ou même philosophiques. Bref, la notion d'objet esthétique comprend les divers aspects de l'œuvre littéraire mais toujours sous forme de concrétisation.

Quelle est donc la différence entre qualité et valeur chez Ingarden ? En quelle mesure la qualité suscite-t-elle une valeur, ou en quel sens la valeur dépend-elle de la présence dans l'œuvre de ce qu'Ingarden appelle une qualité? Il faut dire d'abord que l'esthétique d'Ingarden se définit par une disposition à plaire ou à susciter chez le lecteur un désir de mieux connaître l'objet du désir ${ }^{4}$. Il faut toutefois rappeler et que ce plaisir n'est pas passif en le sens qu'il exige

3. Le mot « unique » veut dire ici que chaque concrétisation réalisée, soit par différents lecteurs, soit par un même lecteur mais à différents moments, est une concrétisation particulière qui ne se répète jamais. Evidemment il y a souvent concordance entre différentes concrétisations selon les possibilités que l'ceuvre présente à ses lecteurs et selon les capacités des ces derniers.

4. Dans ce contexte nous sommes de l'avis que « plaire » et « susciter un désir » sont des synonymes. Ingarden s'intéresse à l'effet (une analyse formelle approfondie) de cette émotion plutôt qu'à sa source psychologique dans l'individu. Ces termes sont néanmoins importants dans sa philosophie parce que leurs signifiés nous renseignent sur d'autres notions, en particulier, la notion d'idée mètaphysique. C'est dire que la notion de plaire au lecteur semble suggérer une esthétique contemplative et classique, tandis que susciter un désir implique plutôt une esthétique de volonté et de puissance. Ces deux notions se résument sous les rubriques « classique » versus « romantique». On se rappelle que selon René Wellek la philosophie d'Ingarden est « classique » (Four Critics: Croce, Valéry. Lukács, and Ingarden, Seattle, University of Washington Press, I98I p. 70); mais on pourrait également lui reconnaître des caractéristiques romantiques (cf. C.J. Rzepka, "Thomas de Quincey and Roman Ingarden: The Phenomenology of the "Literature of Power" », Analecta Husserliana, 33, 199I, p. I19-130; ou bien V. Kocay, "L'Objet esthétique chez Roman Ingarden : prémisses et "valeurs" », Revue Canadienne de Littérature Comparée, 17, 1990, P. 36-44). 
la participation du lecteur, et qu'il n'est pas subjectif, dans le sens d'une rêverie, parce que dans la plupart des cas il exige aussi un effort intellectuel d'interprétation.

Cependant, Ingarden ne prétend pas pouvoir résoudre définitivement la question des valeurs esthétiques. À maintes reprises il suggère même, peutêtre seulement de façon thétorique, qu'il faut encore beaucoup de recherches dans ce domaine avant de pouvoir bien comprendre le lien entre l'œuvre d'art et les valeurs esthétiques que l'œuvre suscite chez le lecteur. Les valeurs sontelles, par exemple, objectives et appartenant à l'œuvre, ou bien subjectives, c'est-à-dire « créées » par le lecteur ? Et cette question en suscite deux autres, à savoir que veulent dire les termes d'objectif et de subjectif sous la plume d'Ingarden? Et puis, en quelle mesure Ingarden parle-t-il de l'intersubjectivité lorsqu'il traite du problème des valeurs esthétiques? Il faut pourtant signaler qu'Ingarden n'emploie le terme « intersubjectif » que fort rarement, et toujours dans le contexte de la sémantique plutôt que dans celui de l'axiologie. Il n'est d'ailleurs pas nécessaire de reproduire ici toute l'ontologie d'Ingarden, la définition de l'objectif de Fink citée plus haut répondant suffisamment à nos besoins. Il convient plutòt de se pencher sur la notion de valeur esthétique car c'est dans cette perspective que se dessine le sens de ces termes chez Ingarden.

\section{La valeur esthétique}

Il est nécessaire de préciser la pensée d'Ingarden à ce sujet afin de pouvoir établir subséquemment la pertinence de la notion de valeur dans les domaines littéraires et esthétiques. Ce n'est pourtant pas une tâche facile. La pensée d'Ingarden eu égard aux valeurs esthètiques est exprimée en différents endroits sous différentes formes. Qui plus est, cette partie de sa philosophie pour intéressante qu'elle soit, donne toujours l'impression d'être seulement ébauchée. Elle est de caractère schématique par rapport à d'autres notions dans sa philosophie, telle la concrétisation et la forme stratifiée de l'œuvre d'art.

En principe l'argument d'Ingarden se présente sous deux formes différentes. I) Selon la première formulation, en trois étapes, l'œeuvre d'art ou l'objet d'art (en opposition avec l'objet esthétique) n'est qu'un objet schématique ou un «squelette » sans valeur esthétique propre : il est neutre dans la perspective des valeurs esthétiques. Les qualités du squelette (également neutres par rapport aux valeurs esthétiques) représentent pourtant la source des qualités d'une valeur esthétique (ästhetisch relevante Qualitäten ${ }^{5}$ ), lesquelles constituent ensuite ce qu'Ingarden appelle la qualité-de-valeur (Wertqualität) de

5. À notre sens le terme «ästhetisch relcuante Qualitäten » (p. 143) est synonyme et du terme «ästhetisch valente Qualitäten » (p. 144) et du terme «ästhetisch wertvollen Qualitäten » (Erlebnis, p. I64). 
l'œuvre, ou la qualité-de-valeur esthétique de l'œuvre (die ästhetische Wertqualität); et cette dernière se fait connaître dans l'objet esthétique formé, lui, dans une concrétisation (Erlebnis, p. I43). 2) Ailleurs, Ingarden dit qu'il existe non pas trois mais quatre groupes différents de qualités (ce qu'il appelle aussi des moments (Momente), à savoir r. les moments neutres de façon axiologique, 2 . les moments qui ont une valeur artistique (künstlerich wertwolle), 3. les moments d'une valeur esthétique et 4. les moments d'une valeur [esthétiquel avec ses déterminations qualitatives (Erlebnis, p. Igo).

Selon le premier schéma il existe trois sortes de qualités :

I) les qualités dépourvues de valeur esthétique ${ }^{6}$;

2) les qualités qui possèdent une valeur esthétique?;

3) la qualité-de-valeur-esthétique ${ }^{8}$;

et, selon le deuxième schéma, il existe 4 sortes de qualités :

I) les qualités neutres de façon axiologique9;

I') les qualités qui possèdent une valeur artistique ${ }^{\text {IO }}$;

2) les qualités qui possèdent une valeur esthétique ${ }^{\mathrm{II}}$;

3) les qualités d'une valeur avec ses déterminations ${ }^{12}$.

Malgré l'étape supplémentaire du deuxième schéma, les deux hiérarchies sont pourtant semblables, ce que nous avons cherchè à faire ressortir en numérotant chaque étape des deux schémas de I) à 3), et en appelant I') l'étape supplémentaire du deuxième schéma. Chaque schéma fait connaître la distinction entre l'œuvre (un objet neutre) et l'objet esthétique (la concrétisation), et chaque schéma traduit bien, nous semble-t-il, la progression, en ce qui concerne les valeurs esthétiques, qu'un lecteur réalise lorsqu'il « concrétise » une œuvre littéraire ${ }^{13}$.

\footnotetext{
6. Asthetisch wertneutrale Qualitäten.

7. Asthetisch relevante Qualitäten

8. Asthetische Wertqualitäte.

9. Axiologisch völlig neutrale Momente.

IO. Künstlerisch wertuolle Momente.

II. Asthetisch wertuolle Momente. Le mot « qualités » est donné ici au pluriel parce que, selon Ingarden, dans l'objet esthétique une qualité d'une valeur esthétique I’apparaît jamais seule mais dans une multiplicité (Mamigfaltigkeit) de qualites liées d'un côté à l'œuvre et de l'autre côté à d'autres qualités d'une valeur esthétique, ce qui donne lieu à de nouvelles qualités, et ainsi de suite.

12. Werte selbst mit ihrer qualitativen Determination

13. Cette progression est en effet hégélienne. Le lecteur est d'abord attiré par l'œuvre, ou bien il désire connaître les qualités qui l'ont attiré. Ensuite il fait l'analyse de
} 
Pour dangereux que soit une telle entreprise nous présentons ici un schéma simplifié de la pensée d'Ingarden afin de rendre notre discussion plus claire. En supprimant les termes « non-essentiels » nous dérivons le schéma suivant :

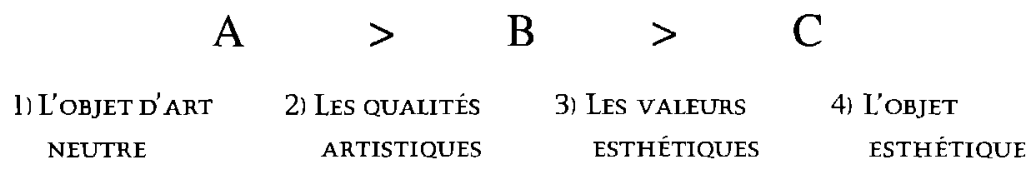

Vu que ce schéma des valeurs représente une progression, c'est la progression elle-même (marquée $\langle A\rangle B\rangle C »$ ) qui attire notre attention. De plus, étant donné que la lecture d'une œuvre particulière donne sans doute une idée plus confuse de la valeur de l'œuvre, les qualités et les valeurs y étant moins nettement démarquées que dans des schémas théoriques, il convient de considérer les valeurs dans la perspective de leur réalisation. C'est-à-dire que l'experience du lecteur aura sans doute tendance à effacer ou à obscurcir l'écart entre les quatre moments de notre schéma. Autrement dit, comment passe-ton A) de l'objet neutre aux qualités artistiques et, B) des qualités artistiques aux valeurs esthétiques qui constituent C) l'étape finale du processus de la concrétisation, la formation de l'objet esthétique?

Ad A) Le squelette neutre de l'cuuvre littéraire comprend certaines qualités, telle la stratification de l'œuvre, la structure quasi-temporelle de l'œuvre (ou la séquence des parties de l'œuvre), et certaines structures catégorielles (kategoriale Strukturen) du monde présenté (Erlebnis, p. I82). Pourtant, certaines de ces qualités « neutres » ont une valeur artistique dans la mesure où elles participent à la constitution des qualités ayant une valeur esthétique. Ainsi Ingarden passe du squelette de l'œuvre dépourvu de qualités esthétiques aux qualités possédant une valeur esthétique (bien que cela soit présenté de façon vague). D'après lui, il est difficile de déterminer quelles qualitès possèdent une valeur esthétique et lesquelles n'en ont pas. Cette

l'cuvre dans la perspective esthétique (ou pré-esthétique selon la terminologie d'Ingarden - c'est dire une analyse du squelette neutre de l'œuvre), et finalement il en fait une concrétisation où se manifeste une qualité métaphysique, ce qu'Ingarden appelle la concrétisation « d'essencetés idéales 》 (I'CEuure d'art littéraire, p. 249-250). La constitution de l'objet esthétique, une unité (Einheitlichkeit; Erlebnis, p. 196), à partir de nombreuses qualités et valeurs disparates, représente chez Ingarden « ce que Hegel voulait probablement dire lorsqu'il parlait "d'une Idée qui se fait voir dans l'apparition" » (« was Hegel wahrscheinlich im Sinne hatte. als er von der "in der Erscheinung durchleuchtenden Idee" sprach») (p. 196). 
distinction dépend, finalement, de l'œuvre particulière et, à notre sens, elle dépend aussi, alors, de celui ou de celle qui réalise la concrétisation. Et on se rappelle que la concrétisation est « intentionnelle».

Selon l'axiologie d'Ingarden cette distinction permet d'établir d'abord que les valeurs esthétiques ne sont pas dans l'œuvre elle-même en tant que propriétés de l'œuvre (p. 146) ${ }^{14}$. Plutôt elles sont valeurs pour celui qui lit l'œuvre littéraire d'une façon particulière, selon l'attitude esthétique plutôt que cognitive. Réciproquement, en tant qu'objet culturel, l'cuvre rend l'attitude esthétique nécessaire à la constitution de l'objet esthétique : l'objet d'art seul n'est pas suffisant à déclencher une expérience esthétique. Celle-ci exige la participation du lecteur qui désire connaître une telle expérience. Sur le plan de notre schéma, Ingarden fait passer ainsi la détermination des qualités (celles qui possédent une valeur esthetique) du domaine objectif du squelette neutre de l'œuvre au domaine subjectif du lecteur en train de lire une œuvre particulière.

Ad B) Il s'ensuit que quelques-unes des qualités de l'œuvre artistique possèdent également de la valeur esthétique, bien que la détermination de ces qualités dépende de l'œuvre en question. Ingarden ne donne pas de formulation précise de cette étape de son schéma, préférant se référer à l'expérience des artistes et se limiter à quelques commentaires de caractère vague. Il dit que certains artistes, dans la création d'une œuvre importante, réussissent à faire associer des valeurs esthétiques à des qualités artistiques (nous y reviendrons). Selon Ingarden, le fait qu'il soit possible de réaliser une ouvre d'art semble montrer en effet que les valeurs esthétiques sont fondées dans les qualités de l'œuvre.

Dans la philosophie d'Ingarden la distinction entre qualités neutres et qualités ayant une valeur esthétique reprend la distinction essentielle à toute cette philosophie, entre l'objet d'art, une certaine forme et un materiel particulier, et l'objet esthétique, une entité qui a lieu dans la conscience du lecteur mais qui est « inspirée » par l'objet d'art. Bien qu'elle ne soit pas une propriété de l'œuvre, selon Ingarden, la valeur esthétique d'une œuvre littéraire a cependant son fondement ontologique dans l'ceuvre elle-même.

Ad c) Sans tenir compte des qualités de valeur négative ni de leur fonction dans l'œuvre, il nous semble que les qualités possédant une valeur esthétique sont la source des valeurs esthétiques de l'œuvтe. La beauté de l'œuvre dépend ainsi des qualités de l'ceuvre qui possèdent de la valeur esthétique, mais elle dépend aussi des relations entre les qualités, de leur fondation dans l'ceuvre. et de la façon dont d'autres valeurs esthétiques y sont liées. (La pensée

14. « Es steht ausser Zweifel, dass gar keine ästhetisch relevante Qualitäteine Eigenschaft des physischen Fundaments eines Kunstwerks unmittelbar bildet » (Erlebnis, p. I46). 
d'Ingarden est nuancée à ce sujet, comme il convient peut-être à un sujet aussi epineux.) En insistant seulement sur ce qui d'après nous constitue l'essentiel de cette notion, il paraît que, selon Ingarden, les qualités qui déterminent la valeur esthétique de l'œuvre possèdent, pour cette raison, une valeur esthétique. Ingarden : «si une qualité est une détermination qualitative d'une valeur esthétique, cela veut dire qu'elle lla qualitél est l'évidente détermination de la façon dont la qualité ayant de la valeur esthétique possède une valeur »

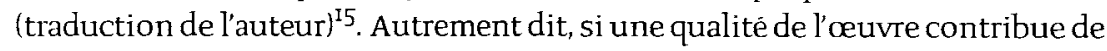
façon claire à la constitution de l'objet esthétique, elle a de la valeur.

Cette formulation, circulaire en ce qui concerne la valeur esthétique de l'œuvre, est également quelque peu mystérieuse. Quelle est finalement la différence entre une qualité ayant une valeur esthétique (ästhetisch relevante Qualität) et une qualité de valeur esthétique (ästhetisch Wertqualität) si ce n'est que le degré auquel la valeur esthétique de l'œuvre représente une entité unifiée et harmonisée. C'est dire que la valeur esthétique (Wertqualität) de l'œuvre semble découler de l'harmonisation des valeurs préalablement dissociées et appartenant à différentes qualités de l'œuvre. Mais nous revenons toujours à la même question, celle de savoir, de quelle façon une qualite possède-t-elle de la valeur ? C'est dire, comment peut-on séparer d'un côté les valeurs des qualités, tout en insistant de l'autre côté sur leur dépendance les unes des autres, et même sur leur identité?

Le caractère vague, voire mystérieux, de cette troisième relation constitutive de l'objet esthétique, fait clairement ressortir, d'après nous, le caractère intuitif des notions de concrétisation et d'objet esthétique. Chaque œuvre particulière possède ses propres qualités et alors ses propres valeurs esthétiques; et chaque concrétisation met en valeur différentes qualités d'une même ceuvre de sorte que l'objet esthétique réalisé doit être lui aussi un objet particulier. Ainsi la concretisation est quelque peu subjective parce que circonscrite en chaque réalisation par le rapport du lecteur à l'œuvre.

Or les termes d'artistique et d'esthétique font problème chez Ingarden, et la formulation que les critiques donnent de la notion d'objet esthétique présente des divergences. Parler de toute la critique est ici impossible. Toutefois, A. Wegrzecki remarque que dans la philosophie d'Ingarden les valeurs esthetiques dépendent des êtres humains et n'apparaissent que dans le domaine des êtres intentionnels ${ }^{\mathrm{t}}$. Dans un article sur la notion de Sujet,

\footnotetext{
I5. «[...] wenn eine Qualität eine qualitative Bestimmung eines ästhetischen Wertes ist, so bedeutet dies, dass sie die eindeutige Bestimmung der Weise ist, wie eine ästhetisch relevante Qualität einen Wert besitzt 》 (Erlebnis, p. I48).

I6. Adam Wegrzecki, « On the Absoluteness of Values »,Joumal of the British Society for Phenomenology, 2, 1975, P. Iog-115; p. Ir3.
} 
article qui compare pour ainsi dire différentes conceptions modernes du Je, J. Fizer conclut que dans la pensée d'Ingarden le Je de la cognition est toujours présent. Sans le sujet, l'objet estéthique tel qu'Ingarden le conçoit ne saurait exister $^{17}$. Pour sa part E. Falk reconnaît le caractère intuitif des valeurs esthétiques, mais semble proposer que l'œuvre d'art constitue à lui seul un objet esthétique, indépendamment du lecteur, et que le lecteur n'est pas la source des valeurs esthétiques, mais ne fait que découvrir celles-ci ${ }^{18}$. Et d'après $M$. Golaszewska la valeur esthétique est une propriété de l'objet esthétique - un objet intentionnel (p. 53) -, et elle est fondée sur la capacité du lecteur/ spectateur de connaître l'objet d'art en une expérience esthétique ${ }^{19}$. Évidemment c'est cette dernière formulation de la notion ingardénienne de valeur esthétique qui nous est la plus utile car elle met clairement en valeur l'importance du lecteur, bien que Golaszewska s'intéresse plus à l'objectivité prétendue de ces valeurs qu'aux conséquences logiques de l'argument d'Ingarden.

L'objet esthétique est pourtant unifié, ou « harmonisé » selon Ingarden. Ce n'est pas le simple mélange de qualités ayant une valeur esthétique. Chez Ingarden c'est une nouvelle entité qui existe chez le lecteur, et il fait connaître une idée métaphysique, laquelle se fait voir (ou entrevoir) lors de l'apparition de l'objet esthétique ${ }^{20}$. La manifestation d'une idée métaphysique dans une conctrétisation représente le point d'aboutissement de l'expérience esthétique dans la philosophie d'Ingarden. Les autres étapes du processus en sont des étapes justement dans la mesure où elles mènent vers ce point final. Dans l'axiologie d'Ingarden ce point final est donc essentiel et nécessaire car il détermine la constitution même des valeurs esthétiques. C'est-à-dire que ce n'est que dans le contexte de la manifestation d'une idée métaphysique, au moyen des qualités et des valeurs de l'œuvre, que les valeurs esthétiques sont justement des valeurs. Autrement dit, la valeur est valeur dans la mesure où elle participe à la réalisation d'une entité harmonieuse que l'on appelle l'objet esthétique et qui manifeste une idée métaphysique.

17. John Fizer, «Deconstruction of the Subject in Light of Ingarden's Epistemology », Reports on Philosophy, 10, 1986, p. 19-26; p. 26.

I8. Eugene H. Falk, « Ingarden's Conception of AEsthetic Values in Literature », Yearbook of Comparative Criticism 10, 1983, p. 79-85; p. 79, p. 83 .

19. Maria Golaszewska, «Aesthetic Values in Ingarden's System of Philosophy », dans P. Graff et S. Krzemien-Ojak (dirs), Roman Ingarden and Contemporary Polish Aesthetics, Warszawa, 1975, p. 47-68; p. 59 .

20. Chez Ingarden le terme «métaphysique» veut dire non pas quelque idee religieuse ou spirituelle dans le sens familier et péjoratif de ce mot, mais plutôt quelque chose qui nous touche, le tragique, le dramatique etc., comme ces émotions se font connaître dans la vie de l'homme. 
C'est pourtant l'importance attribuée à la valeur « harmonisée » de l'objet esthétique qui nous fait rejeter au moins une partie de cette axiologie, c'est-àdire sa linéarité, les valeurs se fondant sur l'objet d'art qui est neutre par rapport à ces valeurs. Or, Ingarden se veut « réaliste ». A son sens la valeur esthétique, bien qu'absolue dans le sens qu'elle ne sert aucun but en dehors d'ellemême là l'encontre des valeurs artistiques qui sont relatives dans le sens qu'elles sont le fondement des valeurs esthétiques), dépend pour ainsi dire d'un objet du monde. Dans le cas d'une œuvre littéraire (mais aussi dans le cas d'une cuvre de musique ou de peinture), il existe un objet indépendant du lecteur/spectateur et cet objet constitue le point d'origine des valeurs esthétiques. Les valeurs ne se réalisent qu'en conjonction avec l'objet. À toute valeur est donc associée une qualité dans l'œuvre, une qualité ayant prècisément de la valeur esthétique en raison de cette association, et cette qualité constitue le fondement ontologique de la valeur qui lui est associée. Ce que nous mettons en question, c'est le rôle du lecteur dans la constitution des valeurs esthétiques. Ingarden semble parfois négliger ce rôle, quoiqu'il ne l'ignore pas tout à fait. D'un même trait nous mettons donc aussi en question la neutralité supposee de l'objet artistique. L'objet artistique, peut-il jamais être véritablement «neutre »?

Il faut tout de suite préciser que ce n'est pas autant l'objectivité des valeurs esthétiques que nous mettons en question ${ }^{21}$. Nous nous intéressons plutôt au point d'origine de ces valeurs dans la philosophie d'Ingarden. En ce sens nous nous demandons quel est le rôle du lecteur dans la formation des valeurs esthétiques par rapport à la fonction exclusive attribuée dans ce but à l'œuvre dans la philosophie d'Ingarden. Toujours soucieux de ne pas transgresser les limites du réalisme, Ingarden sous-estime, à notre sens, la part du lecteur dans la détermination des valeurs esthétiques. Il est toutefois vrai qu'il reconnaît le rôle du lecteur dans la détermination des valeurs, la notion de concrétisation en faisant foi, mais il est tout aussi vrai qu'il hésite à considérer la véritable envergure de cette notion pourtant si capitale dans sa pensée. Le rôle subjectif du lecteur dans la détermination des valeurs esthétiques reste, dit-il, «à déterminer » (Ontologie der Kunst, Ontology of the Work of Art, p. 30g).

Nous sommes pourtant de l'avis que le rôle du lecteur est plus important qu'Ingarden ne le croit; et nous voulons ainsi attirer l'attention sur le côté

2I. Dans deux articles différents Ingarden dresse une liste des sens différents et possibles des mots « objectif» et « subjectif». Ces termes sont polysémiques d'après lui. Sa conception du terme « objectif » comprend les valeurs esthétiques. (Cf. «Das Problem des Systems der ästhetisch valenten Qualitäten », et « Betrachtungen zum Problem der Objektivität », Erlebnis, p. 207-218; p. 219-255.) 
subjectif de la notion de concrétisation. Il découle de la philosophie d'Ingarden que chaque lecteur réalise un objet esthétique particulier, et alors que cet objet est subjectif dans la mesure où il représente la réalisation des valeurs particulières du lecteur ${ }^{22}$. A notre sens, l'harmonie des valeurs esthétiques est impossible sans la participation active du lecteur et sans le désir du lecteur de la réaliser. La réalisation d'une harmonie finale est une notion qui dépasse le fondement artistique de l'œuvre. Pourquoi l'objet esthétique représente-t-il l'harmonisation des différents éléments de l'œuvre et la manifestation d'une " idée métaphysique »? C'est-à-dire, pourquoi l'expérience esthétique doit-elle dépendre d'une perspective téléologique? Ingarden ne le dit pas. Il semble, d'ailleurs, que la notion d'une valeur esthétique culminante présuppose une philosophie de l'homme (une idéologiel qui soit ou bien kantienne ou bien hégélienne. Toujours est-il que l'harmonisation de l'objet esthétique ne peut venir, finalement, que du lecteur, à savoir de sa participation au texte lu.

Le terme d'Abwandlungen ${ }^{23}$ (une modification, une déclinaison ou une conjugaison dans le sens grammatical) dont Ingarden se sert pour parler des qualités ayant de la valeur esthétique (ästhetisch valente Qualitäten) et donnant lieu à des valeurs esthétiques (ästhetische Werte), semble indiquer, d'ailleurs, que l'on peut trouver dans une ouvre littéraire plusieurs qualités d'une même valeur, les valeurs étant par analogie les diverses conjugaisons ou déclinaisons d'une même qualité. Mais pour pouvoir reconnaître les différentes apparitions et les formes variables d'une valeur particulière il faudrait, nous semble-t-il, avoir une idée préconçue de la valeur ainsi représentée. Sinon, on serait incapable de la reconnaittre comme valeur. Dans son œuvre, Ontology of the Work of Art. Ingarden mentionne l'importance de l'expérience esthétique et de la culture générale du lecteur dans la constitution heureuse de l'objet esthétique (p. 230, 238, note 70, 236). Mais si les valeurs esthétiques sont fondées dans l'œuvre artistique, pourquoi faudrait-il un lecteur expérimenté et doué pour les reconnaître? Le besoin d'une telle expérience semble indiquer que les valeurs sont souvent pré-constituées par la société qui entoure le lecteur et l'œuvre d'art analysée, ainsi que par l'histoire individuelle même du lecteur. Ingarden semble ignorer ce fait ou, du moins, il ne le reconnaît pas suffisamment. Cela se voit, d'ailleurs, sur un tout autre plan, celui du noyau de l'objet esthétique.

22. Sur un autre plan il faudrait invoquer les notions de société et d'idéologie qui forment le lecteur, angle sous lequel les marxistes, par exemple, ont attaque la phénoménologie en général, mais cet aspect des valeurs dépasse le cadre de notre discussion. 


\section{Le noyau de l'objet esthétique}

Pour mieux cerner la notion d'objet esthétique il faut invoquer un autre concept explicité dans la philosophie d'Ingarden, cette fois-ci dans son ontologie - celui du noyau d'un objet. Selon l'ontologie ingardénienne tout objet existant possède un noyau qui détermine ou qui constitue les propriétés essentielles de l'objet, et ce noyau reste inchangé malgré les changements physiques que l'objet pourrait subir. Selon Ingarden, même les êtres humains possèdent un noyau qui détermine en partie le caractère de l'individu. Transformer ou altérer le noyau d'un objet ou d'un être humain, c'est détruire cet objet en tant que tel ${ }^{24}$.

Or, vu que l'objet artistique et l'objet esthétique existent, il en découle qu'ils ont chacun un noyau particulier et différent, étant des objets distincts (bien que liés l'un à l'autre de façon ontologique). C'est-à-dire que même si l'existence de l'objet esthétique dépend de l'existence de l'objet artistique, les deux objets existent séparément. Pour déterminer en quoi consiste le noyau de ces deux objets, il faudrait délimiter dans la mesure du possible les propriétés essentielles de l'œuvre littéraire, d’abord dans la perspective artistique et ensuite dans la perspective esthétique. (Ces deux perspectives correspondent, d'ailleurs, aux attitudes théoriques et esthétiques qui caractérisent chez Ingarden la différence entre l'œuvre scientifique et l'œuvre littéraire et esthétique.)

Dans le cas de l'objet artistique il est relativement facile de cerner son noyau. L'cuure littéraire constituée de phrases juxtaposées les unes aux autres et établissant ainsi un contexte ou un référent est indejendante de son auteur. Une fois réalisée l'œuvre littéraire est dissociée des états mentaux et des désirs de son auteur, et comme l'œuvre ne coïncide pas non plus avec les états mentaux du lecteur, ce n'est ni chez le lecteur ni chez l'auteur qu'il convient de chercher le noyau de l'objet artistique. On constate plutôt que tout changement voulu sur le plan de l'œuvre requiert un changement de la forme linguistique de l'œuvre, tout comme un changement de la structure ou de la séquence des phrases entraine une transformation de l'œuvre. C'est dire qu'à notre sens le noyau de l'objet artistique est la forme linguistique de l'œuvre ${ }^{25}$.

24. Cf. Der Streit um die Existenz der Welt, Bd. I, K.5, Tübingen, Max Niemeyer Verlag, Ig64.

25. Dans Ontology of the Work of Art Ingarden précise que la strate de l'œuvre la plus importante est celle du monde représenté. C'est au niveau de la représentation que, selon Ingarden, les valeurs esthétiques sont réalisées. Il faut pourtant se rappeler que la représentation dépend des couches linguistiques de l'œuvre (phonétique et phrastique), sans lesquelles l'œuvre n'existe pas. 
Le noyau de l'objet esthétique est plus difficile à cerner surtout parce que d'après nous Ingarden se trompe dans son explicitation de cette notion. C'est surtout sur le plan du noyau, d'ailleurs, que la faiblesse de l'ontologie ingardénienne des valeurs est mise en relief. Selon Ingarden le noyau de l'objet esthétique est constitué des qualités de l'œuvre artistique, qualités qui attirent l'attention du lecteur. Ingarden : « Dans leur totalité les qualités matérielles et les moments formels de l'œuvre constituent, dans une ceuvre concrétisée, ce que j'appelle le noyau de valeur esthétique de l'objet esthétique » (Traduction de l'auteur ${ }^{26}$. Il distingue alors les qualités de l'objet artistique des valeurs esthétiques qui se réalisent dans une concrétisation. Il ne prétend pas pouvoir dissocier complètement les qualités de l'œuvre des valeurs esthétiques; au contraire, la formule «qualités-de-valeur esthétique » que l'on donne dans la traduction française de son cuvre, Das literarische Kunstwerk ${ }^{27}$, témoigne de l'interdépendance de l'œuvre artistique et de sa concrétisation. Autrement dit, une qualité suscite une valeur, une valeur implique une qualité. Selon la philosophie d'Ingarden le lecteur est attiré par des qualités qui impliquent ou qui suscitent certaines valeurs correspondantes. Bref, le noyau de l'objet esthétique se trouve sur le plan des qualités de l'œuvre. Selon Ingarden, les propriétés inchangeables de l'objet esthétique sont des qualités d'ordre artistique ou technique parce qu'elles appartiennent à l'œuvre artistique. A notre sens cette idée fait savoir, encore une fois, qu'Ingarden, toujours soucieux de ne pas suivre l'exemple dit idéaliste de Husserl, a voulu avant tout maintenir une position « réaliste » en esthétique, ainsi fondant l'objet esthétique sur l'œuvre elle-même.

Ả notre sens cette conception du noyau de l'objet esthétique est erronée, car il en découle que le noyau de l'objet esthétique est situé sur le plan des qualités de l'œuvre. Accepter l'argument d'Ingarden, c'est placer le noyau de l'objet esthétique en dehors de son être puisque l'objet esthétique se réalise dans une concrétisation, c'est-à-dire chez le lecteur. Or, comment peut-on prétendre fonder le noyau d'une concrétisation sur les qualités de l'œuvre (même si ces qualitès sont concrétisées) ${ }^{28}$ ? C'est-à-dire, comment peut-on fonder le noyau esthétique de l'œuvre en dehors de la concrétisation? Il faudrait plutôt le situer sur le plan de la concrétisation elle-même, et à notre sens alors, ce sont les valeurs esthétiques qui constituent ce noyau. Nous

26. In ihrer [den materialen Qualitäten sowie den formalen Momenten] Gesamtheit bilden sie in einen konkretisierten Kunstwerk dasjenige, was ich den ästhetisch wertuollen "Kern" des ästhetischen Gegenstandes nenne (.). (Erlebnis, p. 197).

27. Nous préférons dire les « qualitès ayant une valeur esthétique ».

28. Il n'est pas clair qu'il existe une différence entre une qualité et une qualité concrétisée, nuance qui semble, d'ailleurs, relever du solipsisme. Une qualité est toujours qualité pour quelqu'un. 
sommes d'accord avec Ingarden que les valeurs se réalisent en conjonction avec les qualités de l'œuvre; mais à l'encontre d'Ingarden il nous semble que le lecteur est indispensable à la formulation de l'objet esthétique car, en effet, l'ensemble des valeurs préconstituées (ce qu'on pourrait aussi appeler l'horizon d'attente du lecteur) joue un rôle capital dans le jugement esthétique porté sur une œuvre particulière ${ }^{29}$. Certaines qualités sont recherchées en raison de leur valeur esthétique, leur présence étant donc considérée de façon positive, tandis que d'autres qualités restent inconnues ou mal considérées jusqu'à ce qu'un chercheur aventurier les mette, justement, en valeur.

\section{Conclusion : le progrès en axiologie}

Dans ce qui est peut-être le seul endroit où Ingarden parle de nouvelles valeurs esthétiques, de leur réalisation et de leur objet, il attribue à la création des valeurs une espèce de vague mysticisme. Il vaut la peine de citer en entier le paragraphe où il tient compte du rôle de l'artiste dans la création des valeurs esthétiques :

Dans la composition de son œuvre l'artiste prévoit les harmonies possibles des qualités ayant une valeur esthétique. Dans la concrétisation de son œuvre ces qualités doivent mener à la constitution d'une certaine valeur esthétique. Il [l'artistel cherche les moyens techniques nécessaires à la réalisation de l'harmonie des qualités finales qu'il a désirée. A cette fin il choisit des qualités qui en elles-mêmes restent neutres dans la perspective esthétique, comme la couleur, le son, la forme, qualitès qui en elles-mêmes forment le squelette de l'œuvre. C'est dans ce squelette qu'une certaine diversité de qualités ayant une valeur artistique (nous soulignons) est incarnée. Dans la réalisation des conditions subjectives correspondantes à la concrétisation de l'œuvre (c'est-à-dire dans l'accomplissement par un spectateur qualifié d'une expérience esthétique vécue et correspondantel, la fonction de cette diversité de qualités est de faire apparaître, à partir du squelette de l'œuvre, une diversité de qualités ayant de la valeur esthétique (nous soulignons). Ainsi rend-elle également possible la constitution d'une certaine valeur esthétique attribuée à l'œuvre entière (Erlebnis, p. I77. Traduction de l'auteur ${ }^{30}$.

29. Selon A. Szczepanska, une analyse poussée de la notion de qualités artistiques et esthétiques est seulement possible lorsqu'il s'agit de l'analyse d'une cuvre particulière (p. II8), car à son avis, et elle cite Ingarden à cet égard, le système de valeurs qu'Ingarden propose n'est ni objectif ni subjectif, mais représente plutôt une position intermédiaire, la valeur étant d'un côté absolue et de l'autre côté relative au sujet (p. I2I). D'ailleurs, une telle formule prête à confusion. (A. Szczepanska, "Perspectives of the Axiological Investigations of the Work of Roman Ingarden », Joumal of the British Society for Phenomenology, 2, 1975, p. I16-125).

30. Der Künstler sieht bei der Komposition seines Werkes die möglichen Zusammenstimmungen der ästhetische valenten Qualität voraus, die in seinem Werk, bzw. in dessen Konkretisation zur 
Ce passage établit de façon claire que la valeur esthétique est fondée sur l'œuvre. Pourtant, la progression qui nous mène de l'œuvre d'art à l'objet esthétique reste floue et imprécise. Tout en parlant de la création ou de la production d'un objet d'art par un artiste, Ingarden fait glisser son propos vers la réception de l'œuvre d'art réalisée par le spectateur. Ainsi il ne parle que brièvement de la création de l'œuvre et laisse sans réponse des questions importantes. Comment l'artiste prévoit-il, par exemple, la réalisation chez le spectateur des valeurs esthétiques ? Comment peut-il « prévoir » une valeur esthétique qui, quant à sa réalisation, dépend d'une œuvre terminée et indépendante de l'artiste? L'artiste reste une énigme sous la plume d'Ingarden et, par là, le côté individuel, social, et pré-établi des valeurs esthétiques est escamoté dans sa philosophie. Il parait que chez Ingarden le phénomène signifié par le terme « artiste » est synonyme d'intuition, ou de ce qu'on désignait autrefois par le mot " génie », ce qui ne nous aide guère à déterminer dans quelle mesure la valeur esthétique dépend de celui qui concrétise l'œuvre litteraire. Il faut, à notre sens, reconnaître le rôle que joue le lecteur (et l'artiste) dans la constitution des valeurs esthétiques, valeurs qui restent toujours un peu floues, si ce n'est que parce qu'elles changent incessamment, et qui ne réussissent jamais, paraît-il, à se dissocier entièrement de la subjectivité. Si le noyau de l'objet esthétique est bien comme nous le disons, dans la conscience, il faudrait, pour délimiter cet objet et pour comprendre les valeurs esthétiques, s'orienter plus vers la réception de l'œuvre et vers l'idéologie du critique, et moins vers les qualités artistiques, supposées « neutres » de l'œuvre ${ }^{3 \mathrm{I}}$.

A notre sens, pourtant, dire qu'il existe des qualités neutres, c'est appuyer même notre conception des valeurs esthétiques comme relatives au lecteur et à la société, car une qualité «neutre » implique qu'il y a certains éléments de l'œuvre que nous tenons pour non esthetiques ou pour inimportants dans notre concrétisation. Une fois que l'on attribue de la valeur à un élément de

Konstituienng eines bestimmten ästhetischen Wertes führen sollen. Er sucht entsprechende technische Mittel sur Realisierung der von ihm gewünschten qualitativen Zusammenstimmung. Er wählt also solche ästhetisch neutralen Qualitäten, Farben, Klänge, Gestalten, welche das Skelett eines Werkes bilden und in ihm eine gewisse Mannigfaltigkeit kimstlerisch wertvoller Qualitäten verköpen werden, deren Fumktion darauf beruht, bei Erfillung entsprechender subjektiver Bedingungen (d.h. bei einem befähigten Betrachter und beim Vollzug eines entsprechend verlaufenden ästhetischen Erlebnisses) in der Konkretisation des Werkes, auf Grund seines Skeletts, eine Mannigfaltigkeit ästhetisch wertvoller Qualitäten zur Erscheinung zu bringen and damit auch die Konstituierung eines bestimmten ästhetischen Wertes zu ermöglichen.

31. Dans un article qui porte sur l'origine de l'œuvre d'art, S. Cassedy remarque qu'Ingarden ne parle que rarement de la création artistique. Il en formule une hypothèse intéressante : «On est porté à se demander si la cause ne serait donc 
l'œuvre, pourtant, cet élément devient qualité. C'est dire, croyons-nous, que les qualités et les valeurs ne diffèrent les unes des autres que dans leur perception. La nôtre n'est pourtant pas une position idéaliste. Tout jugement esthétique exige une explication, à la fin toute concrétisation s'offre au jugement des lecteurs, et ainsi la subjectivité est limitée par l'intersubjectif.

Nous rappelons, pour conclure, que notre étude est fondée sur trois notions dans la philosophie d'Ingarden : la concretisation, la distinction de l'objet artistique de l'objet esthétique, et le noyau de l'objet esthétique. La concrétisation représente une participation au texte qui permet la compréhension et l'interprétation. Elle a lieu chez le lecteur. Il découle de la notion de concrétisation une séparation d'ordre ontologique entre l'objet artistique ou l'œuvre, et l'objet esthétique ou la concrétisation. Puisque ces deux objets sont justement des objets ils possèdent chacun, selon l'ontologie d'Ingarden, un noyau qui comprend ses propriétés essentielles.

Cela implique à notre sens que la valeur esthétique d'une œuvre littéraire est à chercher non pas dans l'œuvre mais dans la réception de l'œuvre, parce que cette valeur se réalise dans une concrétisation. Ce n'est pas que l'œuvre littéraire existe dans la conscience du lecteur, c'est que la valeur esthétique de l'œuvre implique l'appréciation du lecteur. Les qualités de l'œuvre sont des qualités dans la mesure où le lecteur leur accorde de la valeur. Cela veut dire que dans le cas de l'objet esthétique c'est le lecteur qui attribue une valeur esthétique à des configurations sur le plan de l'objet artistique, à une suite de phrases peut-être, ou à une conjonction d'idées que le lecteur croit digne de son attention. Et c'est le lecteur qui est ainsi le siège des valeurs esthètiques.

\section{Département de philosophie}

Université St.-Francois-Xavier

la cause ne serait donc pas à l'intérieur du système, si en effet l'insuffisance de la doctrine ingardénienne sur l'origine et la genèse de l'œuvre d'art ne viendrait pas de la contradiction qu'une telle doctrine présenterait pour l'essentiel de la pensée d'Ingarden » (S. Cassedy, « L'Origine et la genèse de l'œuvre d'art chez Ingarden », dans M.E. Kronegger, (dir.), Phénoménologie et littérature, Colloque de Cerisy - 1985 , Sherbrooke, Editions Naaman, 1987, p. 24-42; P. 26). 